\title{
La fusion cellulaire
}

Fabienne Paumet

Le phénomène de fusion entre deux cellules est-il normal?

La fusion cellulaire joue un rôle clef dans de nombreux processus biologiques et pathologiques. Cet événement marque même le début de la vie puisque la fusion d'un spermatozoïde et d'un ovule est à l'origine de tout embryon. Par la suite, ce mécanisme devient fondamental au cours du développement. Chez les mammifères, les cellules du trophoblaste fusionnent entre elles pour former le placenta $(\rightarrow) \mathrm{m} / \mathrm{s} \quad(\rightarrow)$. Au cours du dévelop$1999, \mathrm{n}^{\circ} 11$, pement, les ostéoclastes p. 1236 qui participent à la formation des os résultent de $(\rightarrow) \mathrm{m} / \mathrm{s}$ 2001, $n^{\circ} 12$, p. 1260 la fusion de précurseurs d'origine monocytaire $(\rightarrow)$, et la formation des fibres musculaires de celle des myoblastes durant la myogenèse [1]. La compréhension des mécanismes de fusion entre deux cellules présente des intérêts multiples. II est intéressant de noter que la fusion des cellules musculaires a lieu même chez l'adulte. Ainsi lors d'une lésion musculaire, les cellules satellites prolifèrent et les mécanismes de fusion entre les myoblastes permettront la reconstitution de fibres multinucléées (voir l'Encadré de V. Mouly, p. 696 de ce numéro). On peut dès lors imaginer l'intérêt que peut présenter le contrôle de ce mécanisme dans le cadre d'une thérapie. La fusion de myoblastes génétiquement modifiés avec les cellules musculaires de patients atteints de dystrophie musculaire par exemple, pourrait permettre de reprogrammer les cellules déficientes et de corriger la maladie [2]. La fusion cellulaire s'observe aussi au cours de certaines pathologies, la princi- pale étant l'infection virale. En effet, l'entrée des virus enveloppés (par exemple le virus influenza) dans les cellules nécessite une fusion entre la membrane virale et la membrane de la cellule hôte.

\section{Comment s'effectue}

la fusion de deux cellules?

Cet événement implique le mélange de deux membranes cellulaires en une seule et même bicouche lipidique, aboutissant à la formation d'une large cellule multinucléée dans laquelle les constituants des deux cellules sont partagés. La fusion entre deux membranes, quelle que soit la provenance de ces membranes, requiert deux conditions: (1) surmonter la barrière énergétique créée lors du rapprochement des deux membranes lipidiques chargées et (2) être spécifique. Toute fusion membranaire nécessitera donc l'intervention de protéines capables d'assumer ces deux fonctions: rendre le mécanisme favorable d'un point de vue énergétique, et identifier les membranes à fusionner afin d'assurer la spécificité de la fusion. Notre compréhension des phénomènes de fusion membranaire provient essentiellement des études de fusion entre un virus et une cellule et des études de fusion entre les vésicules intracellulaires et les organites.

La fusion de la membrane des virus avec la membrane plasmique de la cellule hôte fait intervenir une protéine membranaire virale fusogène, comme la protéine hémagglutinine du virus influenza. L'hémagglutinine du virus est responsable de l'adhérence du virus à la membrane, puis de la fusion elle-même, cette dernière étant provoquée par une dimi-
Cellular Biochemistry and

Biophysics Program,

Memorial Sloan Kettering

Cancer Center,

1275 York Avenue, Box 251, New York 10021, États-Unis. paumetf@mskcc.org

nution du $\mathrm{pH}$ induisant un changement de conformation de cette protéine et exposant un peptide de fusion. Les peptides fusogènes sont caractérisés par leur nature amphipathique et leur structure en hélice. Ce peptide s'insère alors dans la membrane hôte, et induit la fusion membranaire [3].

Un mécanisme similaire est utilisé par les protéines SNARE pour catalyser la fusion des vésicules lipidiques avec un organite cible dans la cellule. Les protéines SNARE sont des protéines transmembranaires, localisées de part et d'autre des membranes: les v-SNARE sur les vésicules et les t-SNARE sur les membranes cibles. La fusion aura lieu lorsqu'une v-SNARE reconnaît le t-SNARE qui lui correspond et s'y associe de façon spécifique $(\rightarrow)$. Lors de la formation du complexe SNARE, les deux mem- $(\rightarrow) \mathrm{m} / \mathrm{s}$ 2001, $n^{\circ} 5$, p. 669 leur fusion survient ensuite [4]. Malgré les connaissances accumulées sur les mécanismes de la fusion dans ces deux systèmes, ceux qui régissent la fusion de deux cellules restent très mystérieux. Cependant, on en connaît certains principes: pour que deux cellules puissent fusionner, elles doivent devenir « compétentes», processus impliquant certainement un changement dans l'expression génique et protéique. Par la suite, les cellules doivent se reconnaître et adhérer l'une à l'autre. À la suite de la fusion, un nouveau programme de développement est instauré dans la « nouvelle » cellule hybride.

Différentes protéines contrôlent ce mécanisme de fusion, mais jusqu'à 
présent, très peu ont été activement et directement impliquées dans le phénomène de fusion lui-même. Parmi les candidats, on peut citer les protéines Duf et Sns, impliquées dans la myogenèse chez la Drosophile, ou encore la protéine Bindin intervenant dans la fusion entre l'ovule et le spermatozoïde chez l'oursin. Cependant l'étude de la séquence de ces protéines montre qu'elles ne possèdent pas de peptide de fusion ou de domaine transmembranaire. Les études fonctionnelles confirment que ces protéines semblent jouer un rôle dans un mécanisme d'attraction et de reconnaissance plus que dans le mécanisme de fusion luimême [2]. De meilleurs candidats ont été proposés comme les protéines Fertilin et Meltrin, qui appartiennent à la famille des protéines ADAM (a disintegrin and metalloprotease domain protein) [5]. Les membres de cette famille possèdent en effet toutes les composantes nécessaires pour induire la fusion: un domaine transmembranaire, un domaine d'adhérence et un peptide de fusion putatif. Cependant il n'a toujours pas été clairement démontré que ces protéines sont les fusogènes responsables du mécanisme de fusion.

Très récemment, un candidat plus prometteur a été identifié: la protéine EFF-1. Son gène s'exprime dans les cellules de tous les épithélium juste avant que la fusion n'ait lieu. La protéine $\varepsilon F F-1$ est une protéine transmembranaire comportant, comme tous les fusogènes viraux, une séquence hydrophobe fusogène impliquée dans le mélange des bicouches afin d'induire la fusion. Fonctionnellement, il a été démontré in vivo que la fusion cellulaire était gravement perturbée au cours du développement quand la protéine EFF-1 est mutée [6].

$\varepsilon$ n résumé, même si de nombreux candidats ont été identifiés et même si certains sont très prometteurs, il est nécessaire de confirmer leur implication. Quant au mécanisme de fusion luimême, il reste encore à découvrir.

\section{Pourquoi les cellules}

fusionnent-elles?

La fonction principale de la fusion entre deux cellules serait de reprogrammer leur expression génétique. La fertilisation est un cas extrême de reprogrammation cellulaire puisque dans ce cas, les gamètes haploïdes "se transforment" en une cellule embryonnaire unique diploïde totipotente. La

\section{RÉFÉRENCES}

1. Witze $\varepsilon$, Rothman JH. Cell fusion: an EFFicient sculptor. Curr Biol 2002; 12: R467-9.

2. Taylor M. Muscle development: molecules of myoblast fusion. Curr Biol 2000; 10: R646-8.

3. Colman P, Lawrence M. The structural biology of type I viral membrane fusion. Nat Rev Mol Cell Biol 2003; 4 : 309-19. fusion cellulaire pourrait aussi être une stratégie utilisée par la cellule pour interrompre les contacts entre cellules ou leur migration, prévenant ainsi le déclenchement de signaux ou d'interactions inappropriées. Enfin, la fusion cellulaire pourrait permettre de recruter des cellules vers un tissu afin d'en changer les propriétés originales [1].

Même s'il paraît évident que les cellules peuvent fusionner pour des raisons différentes suivant les circonstances, une conséquence commune semble apparaître: le besoin de reprogrammer des cellules ayant à l'origine une identité distincte. $\nabla$

Cell fusion

\section{NOUVELLE}

\section{Le cerveau adulte: un perpétuel chantier!}

Morgane Lemasson, Pierre-Marie Lledo

Chez l'adulte, le bulbe olfactif est une des rares structures nerveuses soumises au renouvellement permanent d'une catégorie de ses constituants cellulaires: les interneurones. Jusqu'à récemment, on pensait que les étapes cellulaires et moléculaires qui participent au renouvellement des neurones adultes récapitulaient les processus embryonnaires. Dans cette revue, nous distinguerons les processus qui participent à la construction du cerveau (neurogenèse primaire) de ceux qui opèrent dans le cerveau mature (neurogenèse secondaire).
4. Galli T, Martinez-Arca $S$, Paumet F. Mécanisme de la fusion membranaire. Med Sci 2002; 18: 1113-9.

5. Huovila AP, Almeida $\varepsilon$, White JM. ADAMs and cell fusion. Cur Opin Cell Biol 1996; 8: 692-9.

6. Molher W, Shemer G, del Campo J, et al. The Type I membrane protein EFF-l is essential for developmental cell fusion. Dev Cell 2002; 2 : 355-62.
Laboratoire Perception et Mémoire Olfactive, Cnrs URA 2182, Institut Pasteur, 25, rue du Docteur Roux, 75724 Paris Cedex 15, France. pmlledo@pasteur.fr
Les neurogenèses primaire et secondaire

La production de cellules neurales (neurones et cellules gliales) au cours de la vie fœtale constitue une étape primordiale dans la formation du système nerveux central. Une fois produite, la grande majorité des cellules quitte les régions neurogéniques en migrant le long de voies déterminées pour atteindre leurs localisations définitives. La migration des différents 